\title{
Hydrologische und chemische Untersuchungen an den Fließgewässern des Schlitzerlandes. A. Quellen. I. Übersicht
}

von

Jörg BREHM*

\begin{abstract}
SUMMARY
Hydrological and Chemical Investigations of Running Waters of the Schlitzerland (Western Germany). A. Springs, I. Survey.

In a triassic sandstone woodland (Schlitzerland in Hesse, Western Germany) 17 springs were hydrologically and hydrochemically investigated throughout one year (April 1973 - April 1974). Measurements were carried out on water volume, temperature, electrolytical conductivity, $\mathrm{pH}$, alkalinity, chlorinity, dissolved molecular oxygen, dissolved organic material (COD), ammonia, nitrite, nitrate, and free phosphate.

Among the hydrochemical factors investigated the $\mathrm{pH}$-value was closely related especially to the micro-climate in the spring drainage area. In particular the $\mathrm{pH}$ rose both with increasing length of the drainage area and - within the same spring - with decreasing water supply. On the other hand the $\mathrm{pH}$-value diminished with increasing altitude of the drainage area. Simultaneously the highest values were found on SW-slopes and the lowest ones in NE-exposures.
\end{abstract}

\section{EINLEITUNG}

Die Quellwässer, die als zutage tretende Grundwässer aufzufassen sind, können hydrologisch und hydrochemisch als Indikatoren für die Umweltbedingungen in ihren Einzugsgebieten gelten. Sie sollten zugleich Aufschlüsse über den Gesteinsuntergrund, den Boden (einschließlich Vegegation und Nutzung) und das Klima der bodennahen Atmosphäre geben.

Obgleich sich diese frei Generalfaktoren mehr oder weniger wechselseitig beeinflussen, können sie doch örtlich sehr unterschiedliches Gewicht erhalten. Das wird sich besonders deutlich in Quellen mit verhältnismaßig kleinen Einzugsgebieten zeigen; denn hier sollte die Zahl potentiell verschiedener Grundwasserkörper, die sich von einem gemeinsamen (Quell-)Austritt vermischen

\footnotetext{
* Limnologische Flußstation des Max-Planck-Instituts für Limnologie, D 6407, Schlitz
} 
würde, geringer sein — und damit auch die Nivellierung der Grundwassereigenschaften.

Tatsächlich besitzen Quellen mit ausgedehnten Einzugsgebieten vornehmlich allgemeinere, mehr oder weniger gebietstypische Züge. Sie folgen aus den geologischen Formationen, dem Großklima - das seinerseits von der geographischen Lage und der Großmorphologie des umgebenden Landes abhängt und dem Bodenmosaik einschließlich Vegetationsdecke und allgemeiner Nutzung. Vgl. hierzu die zusammenfassende Literatur, u.a. Thurner (1967), Keller (1969), Rössert (1969), Richter und Lillich (1975), Ward (1975) und ganz besonders Matthess (1973).

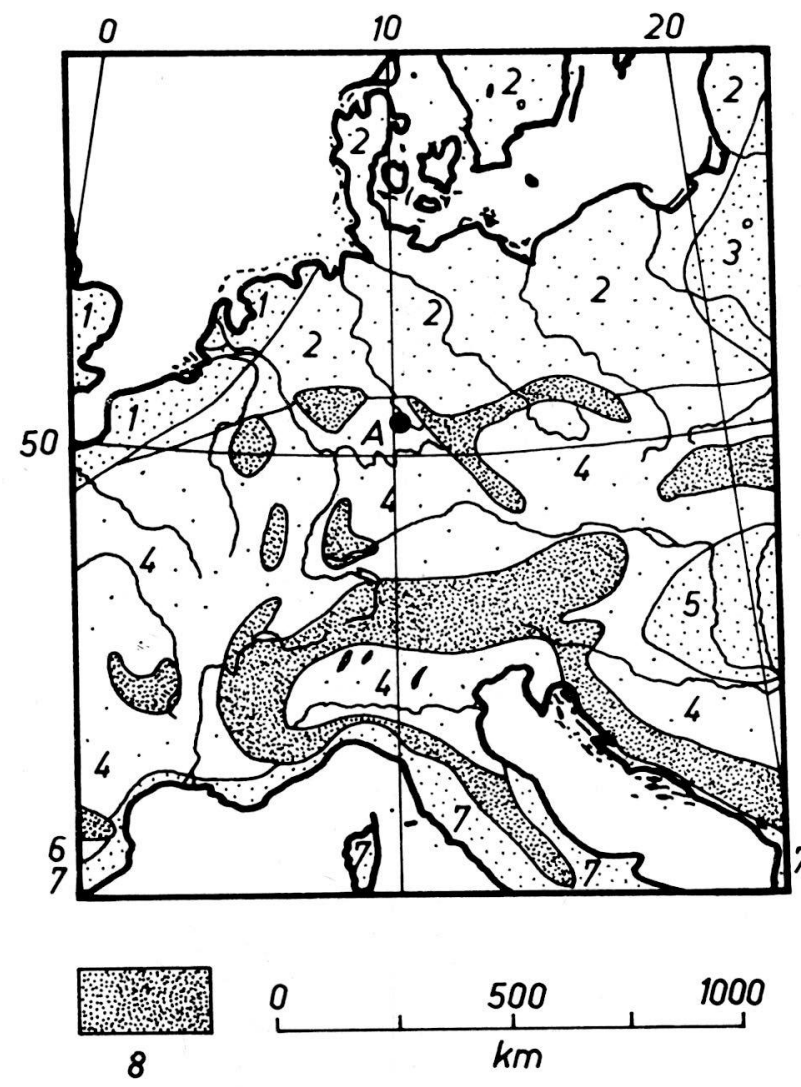

Abb. 1. Die Klimazonen in Mitteleuropa (verändert nach Blüthgen, 1966)

1: Ozeanisches wintermildes, sommerkühles Zyklonalklima; 2: winterkaltes zentraleuropäisches variables Übergangsklima; 3 : mäßig kontinentales sommerwarmes, winterkaltes Klima; 4: sommerwarmes zentraleuropäisches variables Übergangsklima; 5: mäiig kontinentales sonniges sommerheißes, winterkaltes Steppenklima; 6: iberisches wintermildes, sommerheißes Kontinentalklima; 7: sonniges sommerheißes, wintermildes Etesienklima; 8: kühlere, feuchtere und wolkigere Höhenformen der benachbarten Tieflandklimate. A: Lage des Untersuchungsgebietes. 
Die Quellwässer kleiner Einzugsgebiete sind dagegen ortstypischer und in der Tat viel unterschiedlicher. Gut bekannt sind Einflüsse der geologischen Fazies (z.B. durch eingelagerte Salze), der Bodenart einschließlich der natürlichen Vegetation (wie in Bruchwäldern) und der Bodennutzung (etwa durch eingetragene Düngemittel).

Einflüsse des Mikroklimas, das innerhalb einer Großklimazone vom örtlichen Relief - insbesondere der Exposition - abhängt und in enger Wechselbeziehung zu den Böden mit ihrer natürlichen oder auch durch Nutzung veränderten Vegetation steht, sind offenbar bisher nur bei hydrologischen Quellfaktoren - insbesondere der Wassertemperatur - näher bekannt.

Meines Wissens sind Einflüsse des Mikroklimas in den Einzugsgebieten auf

Das Untersuchungsgebiet I

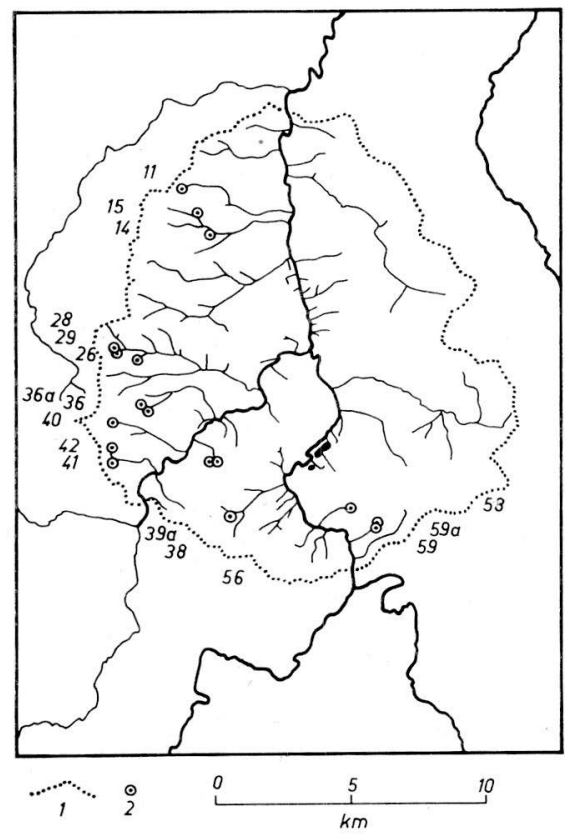

Abb. 2
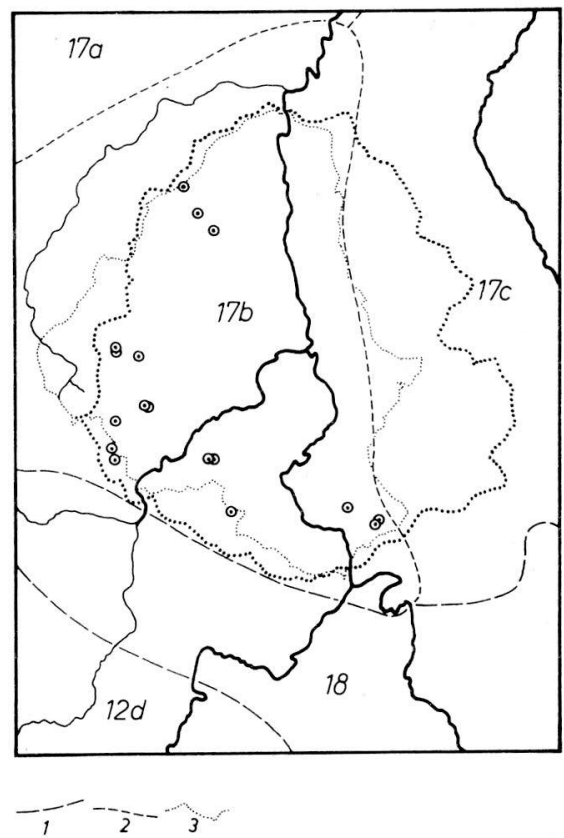

Abb. 3

Abb. 2. Gewässernetz

1: Untersuchungsgebietsgrenzen (Wasserscheiden); 2: untersuchte Quellen. Außerhalb des Untersuchungsgebietes sind nur die Hauptgewässer eingetragen.

Abb. 3. Naturräumliche Zugehörigkeit (verändert nach Rühl, 1967)

1: Grenzen der naturräumlichen Haupteinheiten (Flächensymbole: Zahlen) und 2: der naturräumlichen Untereinheiten (Flächen zusätzlich mit Kleinbuchstaben gekennzeichnet) des Hessischen Berglandes; 3: politische Grenzen des Schlitzerlandes; 12d: Nordöstlicher Unterer Vogelsberg; 17a: Südliches Knüllvorland; 17b: Schlitzer Land; 17c: Vorland der Kuppenrhön; 18: Fuldaer Senke. 
das "chemische Klima" der zugehörigen Quellen zuerst durch den Autor in Buntsandstein-Wald-Ökosystemen - erkannt worden (Brehm, 1974).

Es lag daher nahe, diese Untersuchungen fortzuführen. Dies erschein jedoch erst sinnvoll, nachdem für einige wichtige Wasserinhaltsstoffe, die unter natürlichen oder doch naturnahen Bedingungen in Quellwässern nur spurenhaft auftreten, adäquate quantitative Bestimmungsmethoden verfüfgbar waren. Mit Einführung der Autoanalyzer-Technik war diese Voraussetzung erfüllt.

Die etwas zurückliegenden Quelluntersuchungen wurden nun, in stark erweiterter Form, von 1973 bis 1974 an 17 ausgewählten Gewässern wiederholt. Vierzehn der Quellen waren früher bereits mit untersucht worden; die drei übrigen (zusatzlich mit a gekennzeichnet, vgl. Abb. 2) waren neu aufDas Untersuchungsgebiet II
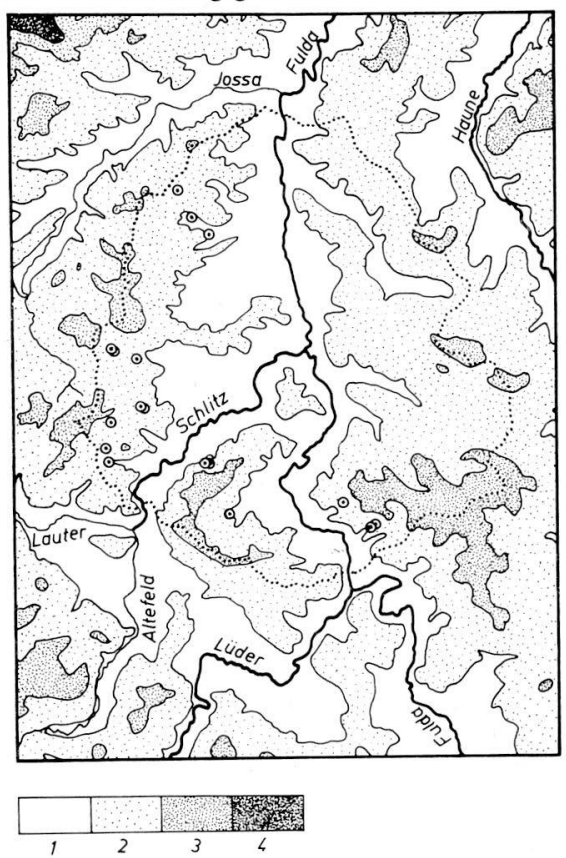

Abb. 4
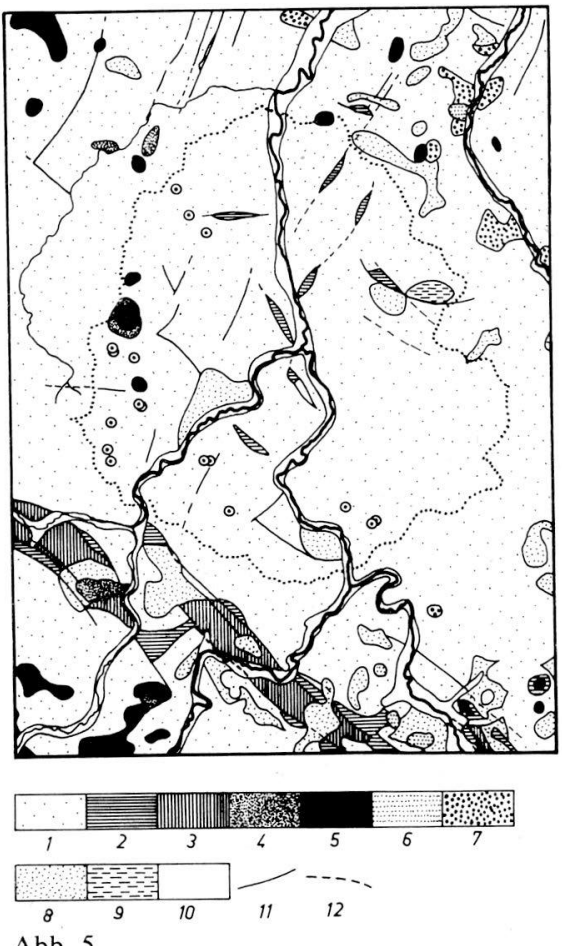

Abb. 5

Abb. 4. Geomorphologie

Höhenschichten (m Meereshöhe): 1: 200-300; 2: 300-400; 3: 400-500 und 4: 500-600

Abb. 5. Geologie (verändert nach Rösing, 1973)

Trias: 1: Buntsandstein; 2: Muschelkalk und 3: Keuper. Tertiär: 4: Sedimente und 5: Magmatische Bildungen (Basalt). Quartär: 6: Präglaziale Bildungen; 7: FluBterrassen; 8: Löß(-lehm); 9: Moorbildungen und 10: junge Hochflutablagerungen; 11: sichere und 12: vermutete Verwerfungen. 
genommen. Die Quellen waren einheitlich nach den folgenden Kriterien ausgesucht.

Das Untersuchungsgebiet III
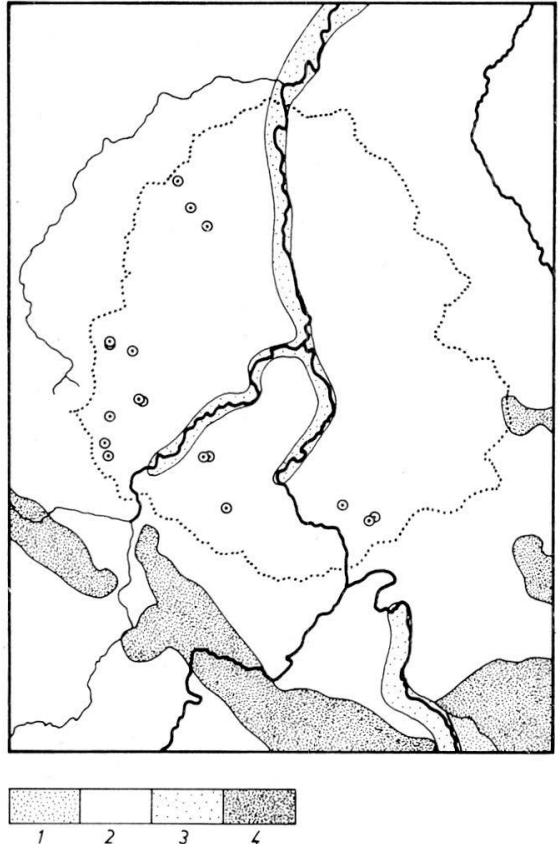

Abb. 6
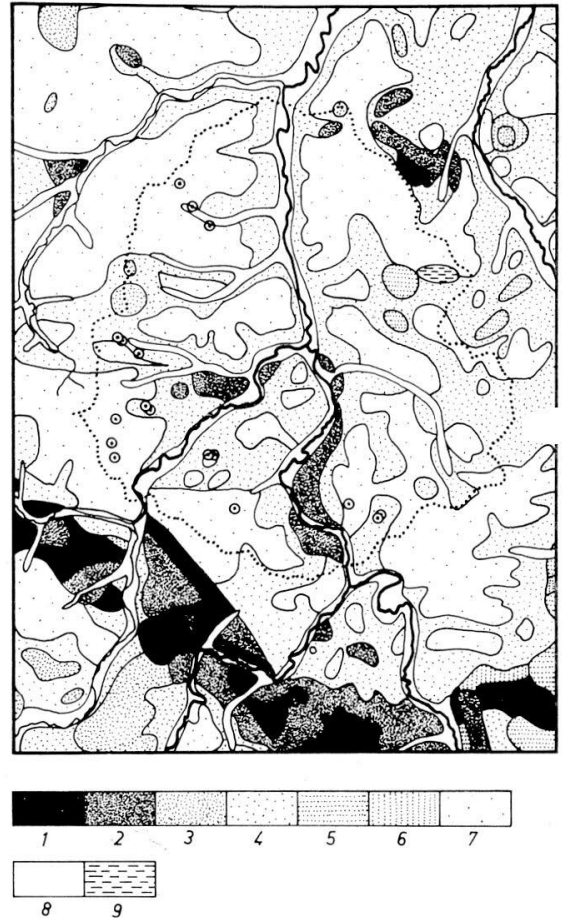

Abb. 7

Abb. 6. Hydrogeologie (verändert nach der Hydrogeologischen Übèrsichtskarte von Hessen, 1955)

1: Kalksteine (Trias); 2: Sandsteine (Trias, auch Tertiär); 3: teilweise verlehmte Sande und Kiese (Quartär); 4: Schiefertone (Trias)

Abb. 7. Böden (verändert nach Schönhals, 195l)

1: Schwerer bis toniger Lehm und Ton (im gesamten Profil kalkhaltige, basenreiche Braunerden, degradierte Rendzinen und Lettenböden); 2: staubsandiger Lehm (überwiegend basenreiche, in der Tiefe kalkhaltige Braunerden = Lößlehmböden); 3: Lehm über vulkanischen Gesteinen, teilweise mit Lößlehm (überwiegend basenreiche Braunerden); 4: teilweise lehmhaltiger Sand, stellenweise auch sandiger Lehm (kalkfreie, meist basenarme, z.T. podsolige Braunerden); 5: staubsandiger Lehm (stark basenverarmte, z.T. podsolierte und gleiartige Braunerden); 6: Lehm bis Ton (basenarme, teilweise gleiartige Lettenböden); 7: teilweise lehmiger Sand (basenarme, mehr oder weniger podsolierte Böden); 8: meist feinsandiger Lehm, auch Sand und Kies, teilweise anmoorig (Aue- und Bruchböden); 9: mehr oder weniger zersetzte Pflanzenreste mit unterschiedlich großen mineralischen Beimengungen (Niederungs- und Ammoore). 
1) Ihr Wasser sollte in mehr oder weniger punktförmigem Austritt ausfließen, nicht etwa linien- oder sogar flächenhaft hervorsickern. 2) Sie sollten perennierend laufen. Indessen fielen die Quellen 36a und 42 später, durch jahrelange zu geringe Niederschläge (vgl. den Monatlichen Witterungsbericht, seit 1971), vorübergehend fast trocken, so daß hier einige Bestimmungen unterbleiben mußten. 3) Ihre Einzugsgebiete sollten geologisch gleich beschaffen sein. Mit Hilfe der geologischen Meßtischblätter Queck, Lauterbach und Das Untersuchungsgebiet IV

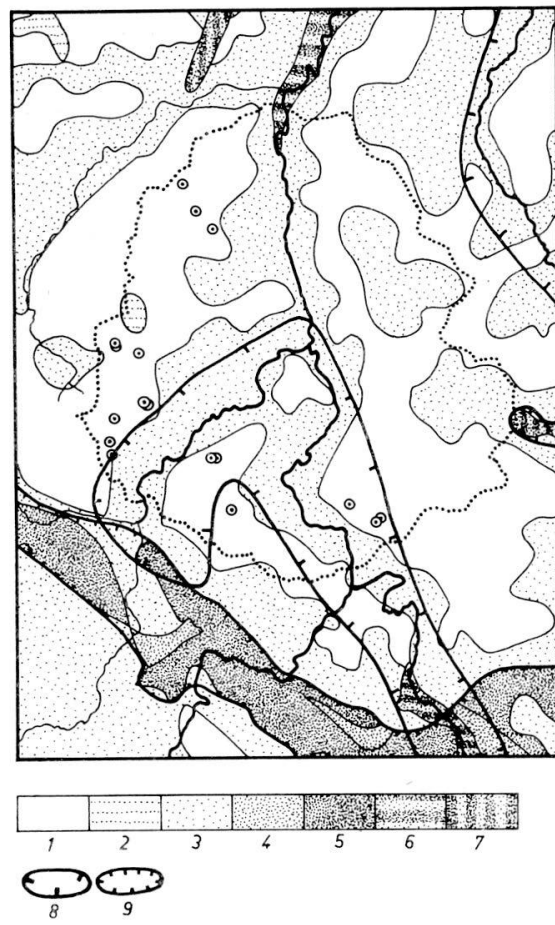

Abb. 8
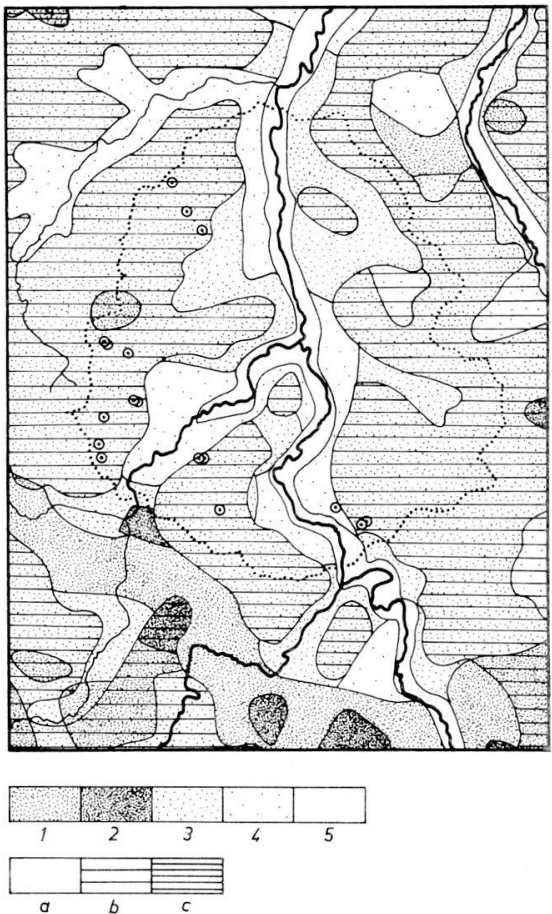

Abb. 9

Abb. 8. Grundwassertypen (verändert nach Thews et al., 1966)

1: sehr weiches, 2: sehr weiches bis weiches, 3: weiches, 4: ziemlich hartes, 5: hartes, 6: mittel- bis ziemlich hartes und 7: mittelhartes, in der Tiefe weiches Grundwasser; 8: versalzenes Grundwasser, in natürlichen Aufstiegen; 9: sulfatreiches Grundwasser, nur örtlich.

Abb. 9. Natürliche Vegetation (verändert nach Knapp, 1954)

A: Natur̈lich vorherrschende Waldgesellschaften (substratbedingt): 1: Berg-Buchen- und Eichen-Hainbuchen-Mischwälder und 2: ihre orchideenreiche, kalkliebende Variante; 3: Eichen- und Buchen-Wälder stark saurer Böden; 4: I und 3 auf engem Raume wechselnd, jedoch 3 überwiegend; 5: Auen- und Quellwälder.

B: Wuchszonen (großklimabedingt): a: Untere und b: obere Buchen-Mischwald-Zone; c: untere Buchenzone. 
Schlitz wurden reine Buntsandsteingebiete ausgewählt. 4) Ihre Einzugsgebiete sollten ferner in der gleichen Form, am vorteilhaftesten forstwirtschaftlich, genutzt sein.

Die Untersuchungen wurden einmal monatlich vorgenommen. Diese Frequenz reichte tatsächlich aus, um den Jahresgang aller berücksichtigten zwölf Gewässerfaktoren für alle Quellen klar herauszuarbeiten.

Der vorliegende Aufsatz kann in die erzielten Untersuchungsergebnisse und ihre Diskussion nur einführen. In den geplanten Folgeaufsätzen sollen die Einzelergebnisse und ausführliche Diskussionsbeiträge hierzu geliefert werden. Da sich die Diskussion mit den Beziehungen der Quelwasser-Eigenschaften zu den Milieufaktoren in den Quelleinzugsgebieten befassen wird, sind Ergänzungsunterschungen traditionell geologischer, geomorphologischer, hydrogeologischer, boden-, vegegationskundlicher und nicht zuletzt mikroklimatologischer Art erforderlich. Sie laufen bereits teilweise.

\section{DAS UNTERSUCHUNGSGEBIET}

Das Untersuchungsgebiet (vgl. Abb. 1 und 4) liegt im Einzugsbereich der Schlitz und des benachbarten Fulda-Abschnittes in Osthessen um 50 40' geographischer Breite und $9^{\circ}$ 30' östlicher Länge in einer Meereshöhe von 200-500 m. Die untersuchten Quellen selbst liegen 270-350 m hoch. Die Grenzen des Untersuchungsgebietes lehnen sich in den Talauen der genannten beiden Flüsse an die politischen Grenzen des Schlitzerlandes an, folgen aber sonst zweckmäßig den Wasserscheiden der Teilniederschlagsgebiete der Schlitzerländer Flußabschnitte (vgl. Abb. 3). Sämtliche untersuchten Quellen, die in Abb. 2 näher bezeichnet sind, liegen jedoch innerhalb des Schlitzerlandes.

Das Untersuchungsgebiet liegt, wie ein Vergleich der Abb. 4-9 zeigt, in einem einheitlichen Landschaftsausschnitt. Geologisch tritt hier der Buntsandstein besonders hervor. Seine mächtigen Schichtenfolgen sind tektonisch in Schollen zerlegt und morphogenetisch in ein Berg- und Hügelland verwandelt und damit landschaftsbestimmend. Entsprechend der weiten Verbreitung auch des anstehenden Buntsandsteins sind die Böden überwiegend sandig, z.T. steinig, und mehr oder weniger lehmdurchsetzt. Sie sind kalkfrei und arm an Basen und den N- und P-haltigen Pflanzennährstoffen. Sie sind daher stark sauer und neigen zur Podsolbildung. Ihre Pflanzenwelt ist artenarm. Die Vegetation besteht unter natürlichen Verhältnissen in den anspruchsloseren LuzulaBuchenwäldern, Traubeneichen-Buchenwäldern und Eichen-Hainbuchenwäldern der Luzula-Subassoziation (vgl. Seibert, 1954). Indessen sind durch den A.nbau von (nicht heimischen) Nadelhölzern, insbesondere der Kiefer (Pinus silvestris), Fichte (Picea abies) und Lärche (Larix decidua und L. kaempferi), und der (einheimischen) Buche (Fagus sylvatica) als wichtigster Laubholzart die (Laub-)Urwälder fast vollständig durch Forstgesellschaften ersetzt.

Der Buntsandstein ist aufgrund seiner Starken Klüftung ein guter Grund- 
Jahreszyklen der untersuchten Gewässerfaktoren I

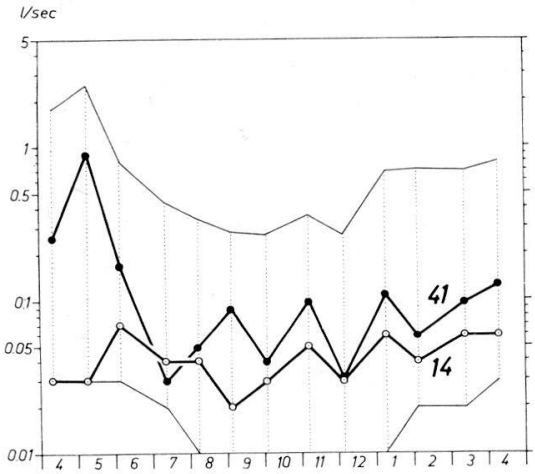

Abb. 10

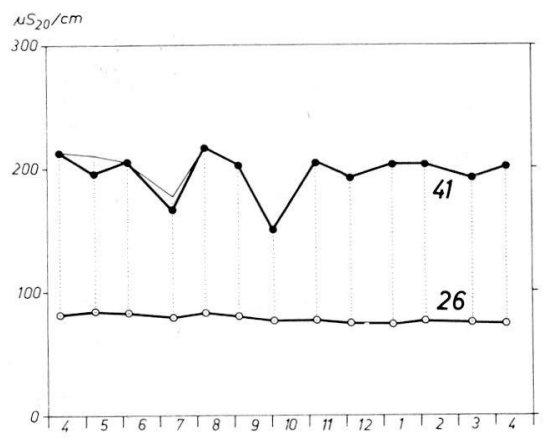

Abb. 12

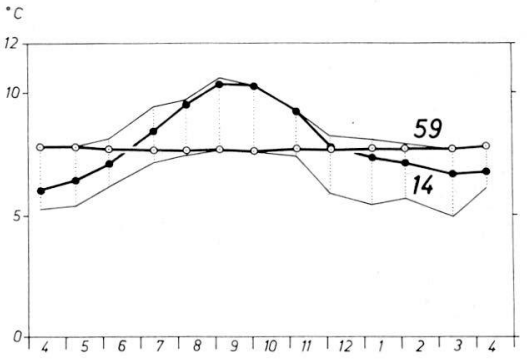

Abb. 11

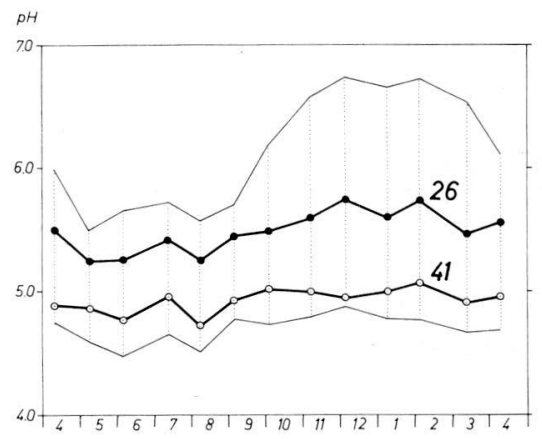

Abb. 13.

Abb. 10. Schüttung

Abb. 11. Wassertemperatur

Abb. 12. Elektrolytische Leitfähigkeit

Abb. 13. pH-Wert

wasserleiter, der aber durch die Einschaltung von wasserundurchlässigen Tonsteinlagen in Stockwerke unterteilt wird, wie häufig ausgebildete Quellhorizonte im Gelände zeigen. Die Einzugsgebiete der Quellen sind verhältnismäßig klein. Bei den 17 untersuchten Gewässern sind sie 12 bis ausnahmswiese 65 ha groß. Sie sind mit den morphologisch leicht abgrenzbaren Niederschlagsgebieten fast deckungsgleich, so daß auch im folgenden beide Begriffe gleichbedeutend nebeneinander verwendet werden können. Wegen der besonderen Gesteins- und Bodenverhältnisse sind im Untersuchungsgebiet überwiegend sehr weiche, recht saure und elektrolytarme Quellwässer zu erwarten. Ins- 
Jahreszyklen der untersuchten Gewässerfaktoren II

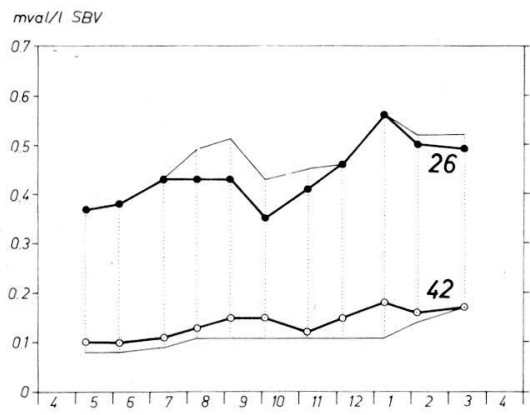

Abb. 14

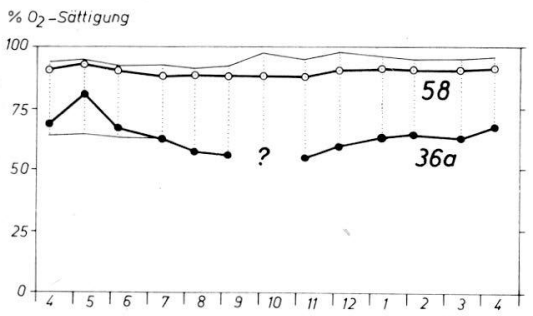

Abb. 16

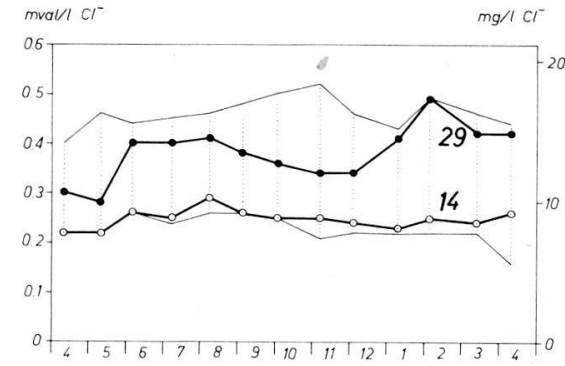

Abb. 15

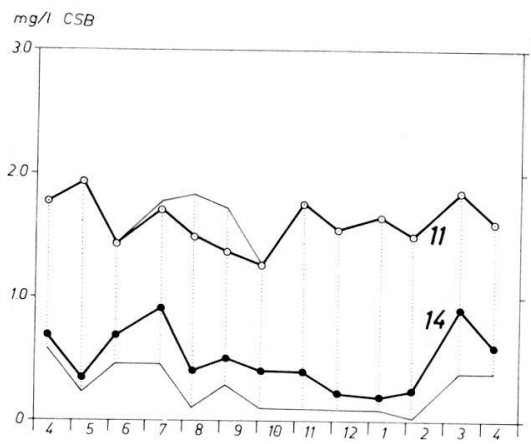

Abb. 17

Abb. 14. Säurebindungsvermögen

Abb. 15. Chlorid-Konzentration

Abb. 16. Sauerstoffsättigung

Abb. 17. Konzentration der gelösten organischen Substanz

besondere sollten die N-Verbindungen (Nitrat) und P-Komponenten (Phosphat) nur spurenhaft auftreten. Vgl. Matthes und Thews (1963), MüllerHaeckel (1965) und Brehm (1974).

Das Untersuchungsgebiet gehört nach der naturräumlichen Gliederung des Hessischen Berglandes von Rühl (1967) zur Haupteinheit Fulda-HauneTafelland (s. Abb. 3). Die untersuchten Quellen liegen danach sogar in derselben Untereinheit Schlitzer Land, das sich erwartungsgemäß nur teilweise mit dem politisch abgegrenzten Schlitzerland deckt.

Großklimatisch ist das Untersuchungsgebiet aufgrund seiner verhältnismaßig meernahen Lage und der vorherrschenden (westlichen) Winde aus den 
Jahreszyklen der untersuchten Gewässerfaktoren III

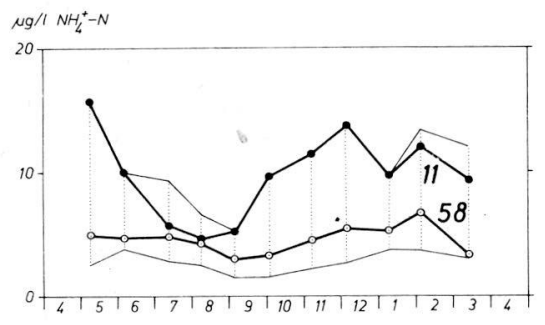

Abb. 18

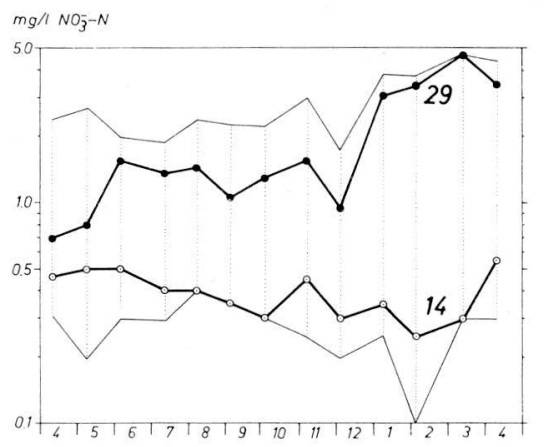

Abb. 20

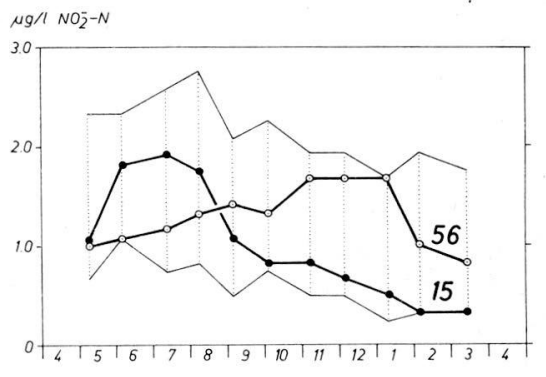

Abb. 19

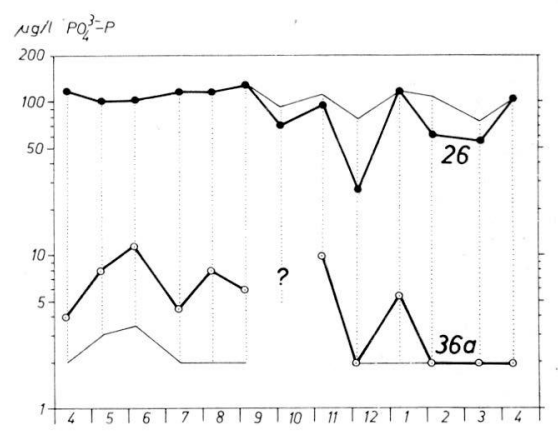

Abb. 21

Abb. 18. Ammonium-Konzentration

Abb. 19. Nitrit-Konzentration

Abb. 20. Nitrat-Konzentration

Abb. 21. Konzentration des gelösten freien Phosphats

nächstliegenden Meeresgebieten in die subozeanische Klimazone (vgl. Abb. 1) einzugliedern. Durch seine Muldenlage zwischen höheren Bergländern und Mittelgebirgen - dem Vogelsberg im SW sowie dem Knüll und dem entfernteren, bedeutenderen Rothaargebirge im NW, die einen Teil der sonst fallenden Niederschläge zurückhalten - ist sein Klima etwas kontinentaler als in seiner Umgebung getönt. Vgl. hierzu Knoch (1950) und die Erläuterungen zum Wasserwirtschaftlichen Rahmenplan Fulda (1964).

Während das Großklima in den Einzugsgebieten der untersuchten Quellen nur aufgrund geringer Unterschiede in der Höhenlage etwas abändert, kann das tatsächliche, örtliche (Mikro-)Klima erheblich variieren. Dies lassen die Unterschiede in der Exposition der Einzugsgebiete sowie in der Artenzusam- 
Der pH-Wert als Funktion der Höhenlage der Quellaustritte

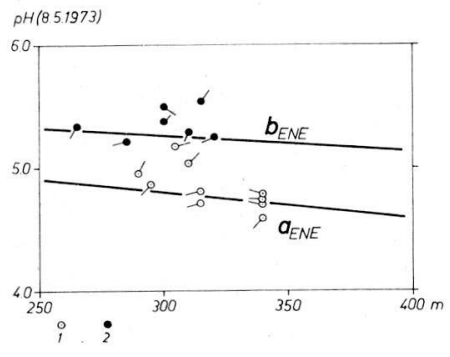

Abb. 22. Frühjahrsverhältnisse

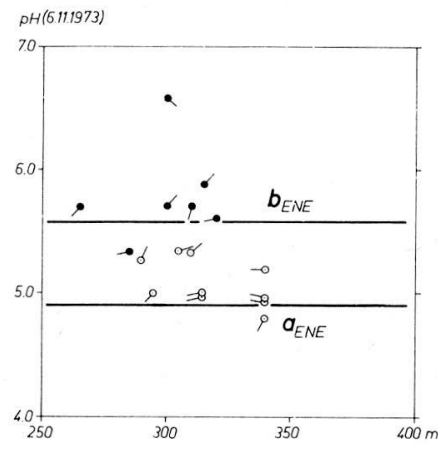

Abb. 23. Herbstverhältnisse

Abb. 22 und 23. 1: Quellen mit kürzeren und 2: mit längeren Einzugsgebieten; a und b: ihre (meereshöhenabhängigen) $\mathrm{pH}$-Gradienten (geltend für Quellen mit ENE-exponierten Einzugsgebieten). Die Pfeile zeigen die Hangneigungsrichtung der Quelleinzugsgebiete an.

Der pH-Wert als Funktion der Hangneigungsrichtung der Quellniederschlagsgebiete

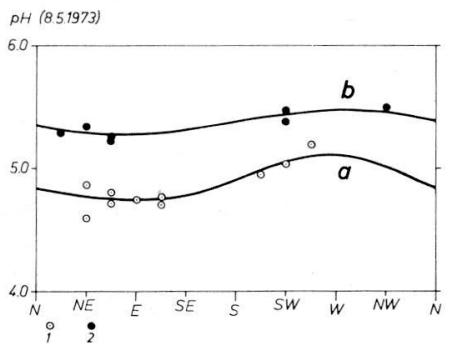

Abb. 24. Frühjahrsverhältnisse

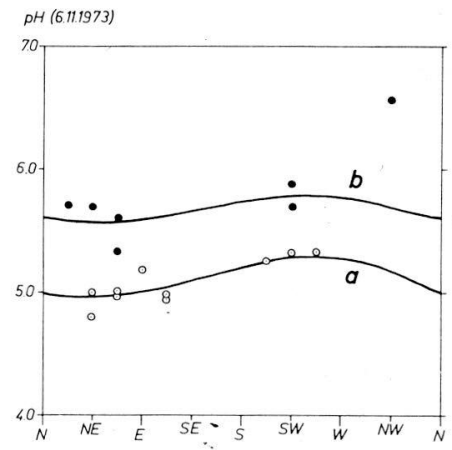

Abb. 25. Herbstverhältnisse

Abb. 24 und 25. 1: Quellen mit kürzeren und 2: mit längeren Einzugsgebieten; a und b: ihre (expositionsabhängigen) $\mathrm{pH}$-Gradienten (geltend für Quellen in $310 \mathrm{~m}$ Meereshöhe).

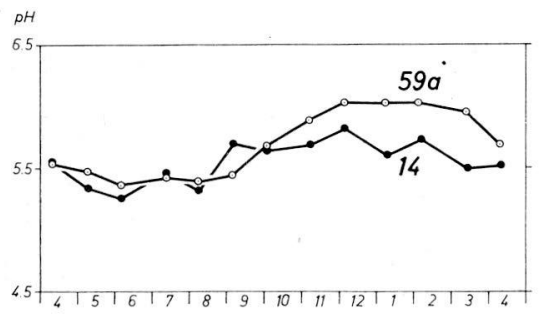

Abb. 26. Der pH-Wert

als Funktion des Witterungsablaufes 
mensetzung und dem Altersaufbau der dort stockenden Wälder erwarten. Vgl. die Lehrbücher der Mikroklimatologie, z.B. Geiger (1961) und Rosenberg (1974).

\section{METHODIK}

Die 17 ausgewählten Quellen wurden vom April 1973 bis zum Vergleichsmonat 1974 einmal monatlich hydrologisch und hydrochemisch untersucht. Dabei wurden die folgenden Gewässerfaktoren berücksichtigt: 1) Schüttung, 2) Wassertemperatur, 3) elektrolytische Leitfähigkeit, 4) pH-Wert, 5) Säurebindungsvermögen (SBV), 6) Chlorid, 7) gelöster molekularer Sauerstoff, 8) gelöste organische Substanz (CSB), 9) Ammonium, 10) Nitrit, 11) Nitrat und 12) freies Phosphat.

(1) wurde mit dem Thomson-Dreieckswehr und (2) mit dem Quecksilberthermometer gemessen. (3) und (4) wurden mit Spezial-Laborgeräten der Wissenschaftlich-Technischen Werkstätten und von Knick - beide Firmen Westdeutschland - ermittelt. (7) wurde manuell jodometrisch, (5) acidometrisch und (6) argentometrisch nach den Deutschen Einheitsverfahren zur Wasseruntersuchung (seit 1960) bestimmt. Im Membranfiltrat (erhalten durch Filtration mit "gewichtskonstanten" Filtern mit $0.45 \mu \mathrm{m}$ mittlerer Porenweite der Membranfiltergesellschaft Göttingen West-deutschland) wurden (8)-(12) vollautomatisch mit dem Autoanalyzer II von Technicon Ireland - nach mehr oder weniger eigenen, speziell angepaßten (noch unveröffentlichten) Verfahren quantitativ bestimmt.

\section{ERGEBNISSE}

Aus Übersichtlichkeitsgründen werden im folgenden für jeden untersuchten Gewässerfaktor nur 2 von jeweils 17 Jahreskurven - darunter eine mit möglichst geringer und eine mit großer Amplitude - und die Gesamtvariationsbreite für die 17 Quellen angeführt (vgl. Abb. 10-21). Gelegentlich kann die Gesamtstreuung nur unsicher angegeben werden, weil einzelne Quellen ( $\mathrm{Nr}$. 36a und 42) zeitweilig nur noch sickerten und sie dann nicht mehr untersucht werden konnten.

Auffällig und zugleich zoophysiologisch bedeutsam erscheint die vergleichsweise hohe Sauerstoffsättigung der Quellwässer im (bewaldeten) Schlitzerländer Buntsandsteingebiet. Sie lag meist über 70\%; nur in Einzelfällen konnte sie bis auf $55 \%$ zurückgehen. Weitere Einzelergebnisse sind den Zeichnungen zu entnehmen.

Im allgemeinen blieb selten wenigstens einer der untersuchten Milieufaktoren in einer Quelle während des Jahres annähernd gleich, wie beispielsweise die Temperatur der Quelle 59 oder die Ammoniumkonzentration in Quelle 58. Häufig traten dagegen ausgesprochen starke Intensitätsschwankungen bei mindestens einem Quellfaktor auf. Vgl. die Schüttung der Quelle 41 oder die 
Nitratkonzentration in Quelle 29. Keine der untersuchten Quellen war in allen erfaßten Gewässerfaktoren gleich veränderlich, wie hier die Quelle 41 zeigen mag. Ihre Wasserführung schwankte im Untersuchungszeitraum zwischen 0.03 und $11 / \mathrm{sec}$ (Verhältnis Minimum: Maximum = 1:33), die Nitratkonzentration zwischen 0.3 und $0.6 \mathrm{mg} / \mathrm{l} \mathrm{N}(1: 2)$ und schließlich der pH-Wert zwischen 4.8 und 5.1 (1:1.06). Insgesamt wiesen die Quellen - obgleich in ihren Einzugsgebieten einheitlich mittlerer Buntsandstein anstand und der Boden ausschließlich forstlich genutzt wurde - eigene, untereinander kaum verwechselbare Muster von hydrologischen und hydrochemischen Eigenschaften vor.

Daneben offenbarten die 17 Quellen jedoch auch gemeinsame Züge: Fünf der erfaßten Gewässerfaktoren (Schüttung, Temperatur, pH, SBV und Ammonium-Konzentration) änderten sich bei allen Quellen jeweils (nahezu) gleichsinnig. Einzelheiten dazu sind den Abb. 10, 11, 13, 14 und 18 zu entnehmen.

\section{DISKUSSION}

Von den vielfältigen Einflüssen der Einzugsgebiete auf die hydrologischen und hydrochemischen Eigenschaften der zurgehörigen Quellen können hier nur die augenfälligsten kurz erörtert werden. Dies soll am Beispiel des Quellwasser-pH-Wertes geschehen.

Nach Villwock (1963) und Meisl (1965) ist insbesondere der mittlere Buntsandstein, in dessen Bereich die Einzurgsgebiete aller untersuchten Quellen liegen, karbonatfrei. Folglich sind auch seine Böden (primär) kalkfrei. Da andererseits seine fast ausschließlich quarzitischen und silikatischen Mineralien sehr schwer löslich, chemisch verwitterbar sind (vgl. Louis, 1968, Ganssen, 1972, Scheffer und Schachtschabel, 1973, sowie Schwarzbach, 1974), verliert das Sickerwasser, das sich im Boden vor allem mit $\mathrm{CO}_{2}$ angereichert hat und wegen der geringen Pufferkapazität stärker angesäuert ist, auch als Grundwasser im Gesteinsuntergrund nur verhältnismäßig wenig Wasserstoff-Ionen an das Untergrundgestein. Der Grundwasser-pH-Wert wird folglich niedrig bleiben, zumal die Pufferkapazität des Grundwassers kaum zunimmt. Grundsätzlich sollte jedoch der pH-Wert mit zunehmender Intensität des Grundwasser-Grundwasserleiter-Kontaktes, bei dem die $\mathrm{H}+-$ Ionen am Gestein sorbiert und eingetauscht werden, steigen.

Bezeichnenderweise lagen tatsächlich die pH-Werte in den Quellen mit den längeren Einzugsgebieten - unter sonst gleichen Bedingungen - höher als in den übrigen (vgl. Abb. 22-25). Darüber hinaus erreichten sie in derselben Que!le während der schüttungsärmsten Jahreszeit ihren Höchststand (vgl. auch Abb. 26). In Quelle 14 änderte sich dabei der pH-Wert - offenbar durch unmittelbare Witterungseinflüsse - kurzfristig. In Quelle 59a waren diese Einflüsse stark gedämpft, so daß ihr $\mathrm{pH}$-Wert eher den Witterungs-Großablauf widerspiegelte.

Das Witterungsgeschehen im Einzugsgebiet beeinflußte indessen nicht nur 
über den Gesteinsuntergrund den pH-Wert des Quellwassers, wie zuletzt angedeutet, sondern auch über die Böden. Vgl. wieder die Abb. 22-25! Unter sonst vergleichbaren Bedingungen im Einzugsgebiet fiel der $\mathrm{pH}$-Wert mit zunehmender Höhenlange der Quelle. In gleicher Meereshöhe traten Tiefstwerte in Quellen mit NE-exponierten Einzugsgebieten auf. Jeweils am höchsten lagen die Quellwasser-pH-Werte an SW-Hängen.

Das Witterungsgeschehen wird im allgemeinen innerhalb eines Klimagebietes mit zunehmender Meereshöhe kühler, feuchter und temperaturausgeglichener ("ozeanischer"). Vgl. hierzu die zusammenfassende mikroklimatologische Literatur, z.B. Geiger (1961), Hartmann und Schnelle (1970), Mitscherlich (1971) und Rosenberg (1974). Parallel dazu sollten - unter sonst vergleichbaren Bedingungen — durch die zunehmend begünstigte Humusbildung die Böden saurer werden - und damit auch die sie passierenden Niederschlagswässer. Ähnlich ist das Mikroklima an N- und NE-Hängen am stärksten ozeanisch und in S- bis SW-Lagen am kontinentalsten getönt. Auch hier sind die niedrigeren Quellwasser-pH-Werte mit dem humideren Klima korreliert.

Der einzelne, extrem hohe pH-Wert in Abb. 23 und 25 deutet auf ein Kalkvorkommen (Muschelkalkreste?) im Einzugsgebiet dieser Quelle (Nr. 38) hin, zumal da gleichzeitig das Säurebindungsvermögen für Buntsandsteinverhältnisse weit überdurchschnittlich erhöht war.

\section{ZUSAMMENFASSUNG}

In einem bewaldeten Buntsandsteingebiet (Schlitzerland in Hessen, Westdeutschland) wurden 17 Quellen während eines Jahresablaufes (April 1973 - April 1974) hydrologisch und hydrochemisch untersucht. Berücksichtigt wurden dabei neben der Schüttung und Quelltemperatur die elektrolytische Leitfähigkeit, pH-Wert, Säurebindungsvermögen, Chlorid, gelöster molekularer Sauerstoff, gelöste organische Substanz (CSB), Ammonium, Nitrit, Nitrat und freies Phosphat.

Unter den chemischen Quellwasserfaktoren ließ der pH-Wert besonders gut Einflüsse durch die herrschenden Umweltbedingungen, insbesondere durch das Mikroklima, im Einzurgsgebiet erkennen. Unter sonst vergleichbaren Verhältnissen stieg der $\mathrm{pH}$-Wert mit zunehmender Länge des Quelleinzugsgebietes und - in derselben Quelle - mit zurückgehender Schüttung. Umgekehrt sank der pH-Wert mit zunehmender Höhenlage des Einzugsgebietes und - in gleicher Meereshöhe von den SW - zu den NE-Hängen.

Für ihre Hilfe danke ich meiner Assistentin, Frau Edith Schäfer, sowie den Herren Egon Gremm und Georg Susemichel.

\section{LITERATUR}

BLÜTHGEN, J. (1966): Europa, Klimagebiete 1:30000000. In: Der große Bertelsmann-Weltatlas: Karten-S. 133 (Gütersloh)

BREHM, J: (1974): Hydrologische und chemische Übersichtsuntersuchungen an den Fließgewässern des Schlitzerlandes. II. Elektrolyte in Quellwässern. - Beiträge zur Naturkunde in Osthessen 7/8: 78-93

Deutsche Einheitsverfahren zur Wasser-, Abwasser- und Schlamm-Untersuchung. 3., völlig neu bearb. Aufl. (seit 1960): Löseblattform (Weinheim) 
GANSSEN, R. (1972): Bodengeographie, mit besonderer Berücksichtigung der Böden Mitteleuropas. - 2., völlig umgearb. Aufl. (unt. Mitarb. von Z. GRACANIN): 325 S. (Stuttgart)

GEIGER, R. (1961): Das Klima der bodennahen Luftschicht. Ein Lehrbuch der Mikroklimatologie.- 4., neubearb. u. erweit. Aufl.: $646 \mathrm{~S}$. (Braunschweig)

Geologische Karte von Hessen 1:25 000, Blatt Lauterbach (1935): Bearb. von O. DIEHL (Darmstadt)

---- Blatt Queck (1963): Bearb. von M. LAEMMLEN (Wiesbaden)

---- Blatt Schlitz (1964): Bearb. von H. G. KUPFAHL (Wiesbaden)

HARTMANN, F. K., und F. SCHNELLE (1970): Klimagrundlagen natürlicher Waldstufen und ihrer Waldgesellschaften in deutschen Mittelgebirgen: $176 \mathrm{~S}$. (Stuttgart)

Hydrogeologische Ubersichtskarte von Hessen 1:600000 (1955): Bearb. vom Hessischen Landesamt für Bodenforschung (Wiesbaden).

KELLER, G. (1969): Angewandte Hydrogeologie: 412 S. (Hamburg)

KNAPP, R. (1954): Natürliche Vegetation und Wuchsräume in Hessen 1:600 000 (Wiesbaden)

KNOCH, K. (1950): Klima-Atlas von Hessen (Bad Kissingen)

LOUIS, H. (1968): Allgemeine Geomorphologie. - Lehrbuch der Allgemeinen Geographie 1: $522 \mathrm{~S}$; 3., neu bearb. u. stark erweit. Aufl. (Berlin)

MATTHESS, G. (1973): Die Beschaffenheit des Grundwassers.- Lehrbuch der Hydrogeologie 2: $324 \mathrm{~S}$. (Berlin und Stuttgart)

MATTHESS, G., und J. D. THEWS (1963): Hydrogeologie. In: LAEMMLEN, M.: Erläuterungen zur Geologischen Karte von Hessen 1:25 000, Blatt Nr. 5222 (Grebenau): S. 245-281 (Wiesbaden)

MEISL, S. (1965): Petrographie der Buntsandsteinsedimente. In: KUPFAHL, H. G.: Erläuterungen zur Geologischen Karte von Hessen 1:25000, Blatt Nr. 5323 (Schlitz): S. 105-122 (Wiesbaden)

MITSCHERLICH, G. (1971): Wald, Wachstum und Umwelt. Eine Einführung in die ökologischen Grundalgen des Waldwachstums. 2. Bd.: Waldklima und Wasserhaushalt: $365 \mathrm{~S}$. (Frankfurt/Main)

Monatlicher Witterungsbericht. Amtsblatt des Deutschen Wetterdienstes (seit 1971): Ab Band 20

MÜLLER-HAECKEL, A. (1965): Ungenutzte Quellwässer und oberirdische Wässer. In: KUPFAHL, H. G.: Erläuterungen zur Geologischen Karte von Hessen 1:25 000, Blatt Nr. 5323 (Schlitz): S. 178-184 (Wiesbaden)

RICHTER, W., und W. LILLICH (1975): Abriß der Hydrogeologie: 281 S. (Stuttgart)

RÖSING, F. (1973): Geologische Übersichtskarte von Hessen 1:300 000.- 2. Aufl. (Wiesbaden)

RÖSSERT, R. (1969): Grundlagen der Wasserwirtschaft und Gewässerkunde: 302 S. (München und Wien)

ROSENBERG, N. J. (1974): Microclimate: The Biological Environment: 315 S. (New York, London, Sydney und Toronto)

RUHL, A. (1967): Das Hessische Bergland. Eine forstlichvegetationsgeorgraphische Übersicht.Forschungen zur Deutschen Landeskunde 161: S. (Bad Godesberg)

SCHEFFER, F., und P. SCHACHTSCHABEL (1973): Lehrbuch der Bodenkunde. - 8., unveränd. Aufl.: $448 \mathrm{~S}$. (Stuttgart)

SCHÖNHALS, E. (1951): Bodenkundliche Übersichtskarte von Hessen 1:300 000 (Wiesbaden)

SCHWARZBACH, M. (1974): Verwitterung und Bodenbildung. In: BRINKMANN, R. (Herausg.): Lehrbuch der Allgemeinen Geologie 1: S. 50-95 (Stuttgart)

SEIBERT, P. (1954): Die Wald- und Forstgesellschaften im Graf Görtzischen Forstbezirk Schlitz. - Angewandte Pflanzensoziologie 9: $63 \mathrm{~S}$. Mit einer farbigen Vegetationskarte 1:15 000 (Stolzenau)

THEWS, J. D., und ein Autorenkollektiv (1966): Übersichtskarte der Grundwasserbeschaffenheit in Hessen 1:300 000 (Wiesbaden)

THURNER, A. (1967): Hydrogeologie: 350 S. (Wien und New York)

VILLWOCK, R. (1963): Zur Petrographie des Buntsandstein. In: LAEMMLEN, M.: Erläuterungen zur Geologischen Karte von Hessen 1:25000, Blatt Nr. 5223 (Queck): S. 134-165 (Wiesbaden)

WARD, R. C. (1975): Principles of Hydrology.- 2. Aufl.: 367 S. (London)

Wasserwirtschaftslicher Rahmenplan Fulda. Erläuterungen (1964): 212 S. (Wiesbaden) 